\title{
Bedside ultrasound measurement of optic nerve sheath diameter in patients with sepsis: a prospective observational study
}

\author{
Ziyue Yang, Cuihong Qin, Shuguang Zhang, Shaohua Liu and Tongwen Sun * (D)
}

\section{Background}

Sepsis-associated encephalopathy (SAE) usually manifests as sleep awakening cycle disturbance, cognitive impairment, delirium, and coma [1]. Considering that brain edema secondary to SAE is one of the complications and causes of death in patients with sepsis, early detection of intracranial hypertension (ICH) is of great significance for timely intervention and improved prognosis. However, most patients with sepsis without intracranial infection have no indications for invasive intracranial pressure (ICP) monitoring; thus, non-invasive ICP monitoring was selected. Bedside ultrasonography measurement of the optic nerve sheath diameter (ONSD) offers a favorable alternative and is presently a new technique [2, 3]. Using this method, we compared the differences in ONSD/ICP between patients with and without SAE, discussed the correlation between ONSD and Glasgow Coma Scale (GCS) score, and evaluated the value of ONSD in predicting the prognosis of patients with sepsis.

\section{Methods}

This study was conducted in the general intensive care unit (GICU) and included patients diagnosed with sepsis from November 2019 to January 2020 [4]. Patients with any of the following criteria were excluded from this study: patients with age $<18$, vitreous hemorrhage, eye surgery, central nervous system (CNS) infection, cerebrovascular accident, brain

\footnotetext{
* Correspondence: suntongwen@163.com

General Intensive Care Unit, The First Affiliated Hospital of Zhengzhou University, Zhengzhou Key Laboratory of Sepsis, Henan Key Laboratory of Critical Care Medicine, Zhengzhou 450052, China
}

trauma, or previous neurosurgery. If the patient had changes in mental state, especially in consciousness and cognition, and excluding other factors that cause mental changes, the physician in charge decided whether to diagnose the patient with SAE. According to this standard, the patients were divided into three groups: non-SAE group, SAE group, and SAE recovery group, and the ONSD was measured within $24 \mathrm{~h}$ of admission. The head of the bed was $30^{\circ}$ above the horizontal line, and the ONSD was measured at the retrobulbar $3 \mathrm{~mm}$ position, accurate to $0.1 \mathrm{~mm}$.

\section{Results}

A total of 142 ONSD ultrasound examinations were performed on 90 patients with sepsis during this trial (nonSAE 71, SAE 51, SAE recovery 20), and the median ONSD in each patient group was $5.1(4.75-5.4) \mathrm{mm}, 5.9$ $(5.6-6.25) \mathrm{mm}$, and $5.35(5.075-5.5) \mathrm{mm}$, respectively (Table 1, Fig. 1e). The ONSD of patients with SAE was significantly wider than non-SAE patients (Mann-Whitney $U$ 395.5, $p<0.001$ ) and SAE recovery patients (Fig. 1a, b). After drawing the receiver operating characteristic (ROC) curve (area under the curve, AUC, 0.894, $p<0.001$ ), we found the best critical value of ONSD for the detection of SAE in patients with sepsis was $\geq 5.5$ $\mathrm{mm}$, with a sensitivity of $80.4 \%$ and specificity of $83.5 \%$ (Fig. 1c). The ONSD showed a significant negative correlation with the GCS score $(\mathrm{rs}=-0.666, p<0.001)$ and the serum albumin level ( $\mathrm{rs}=-0.249, p=0.003$ ). Additionally, we found a correlation between ONSD and the 


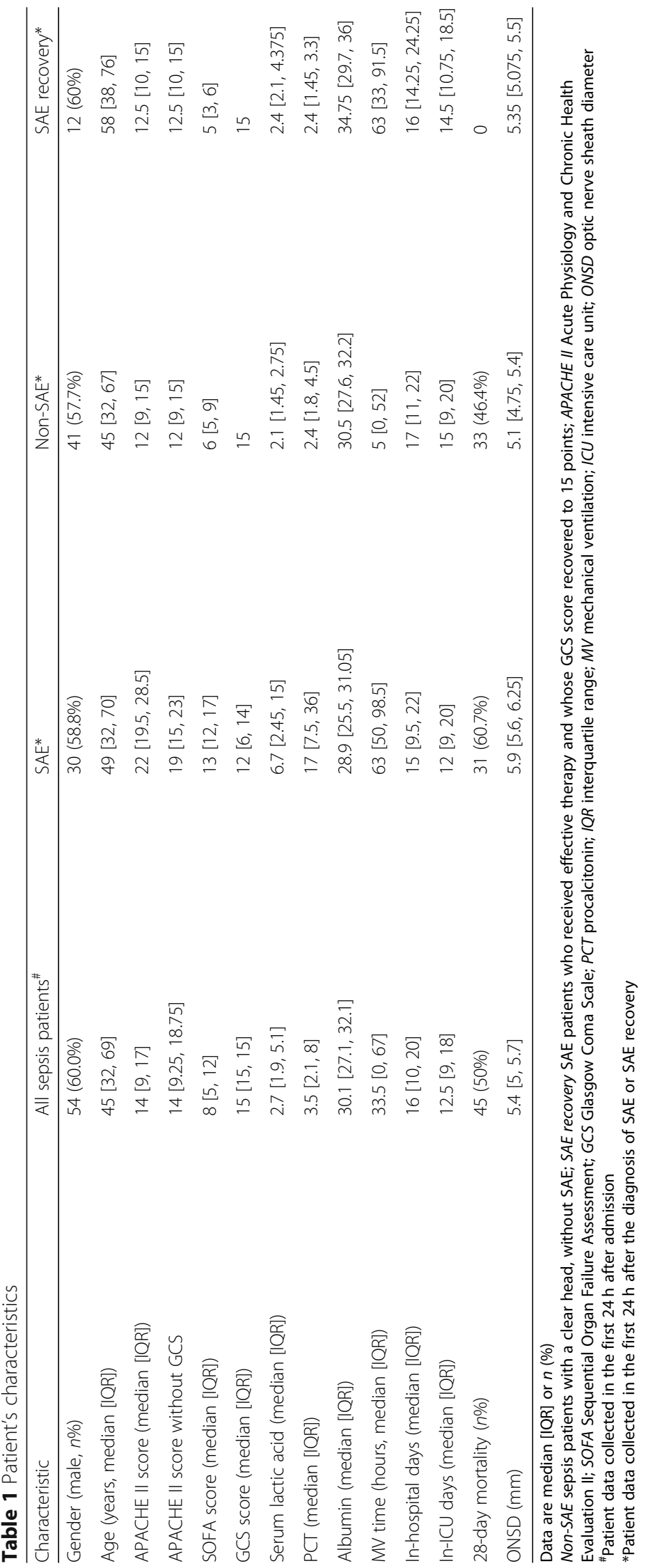



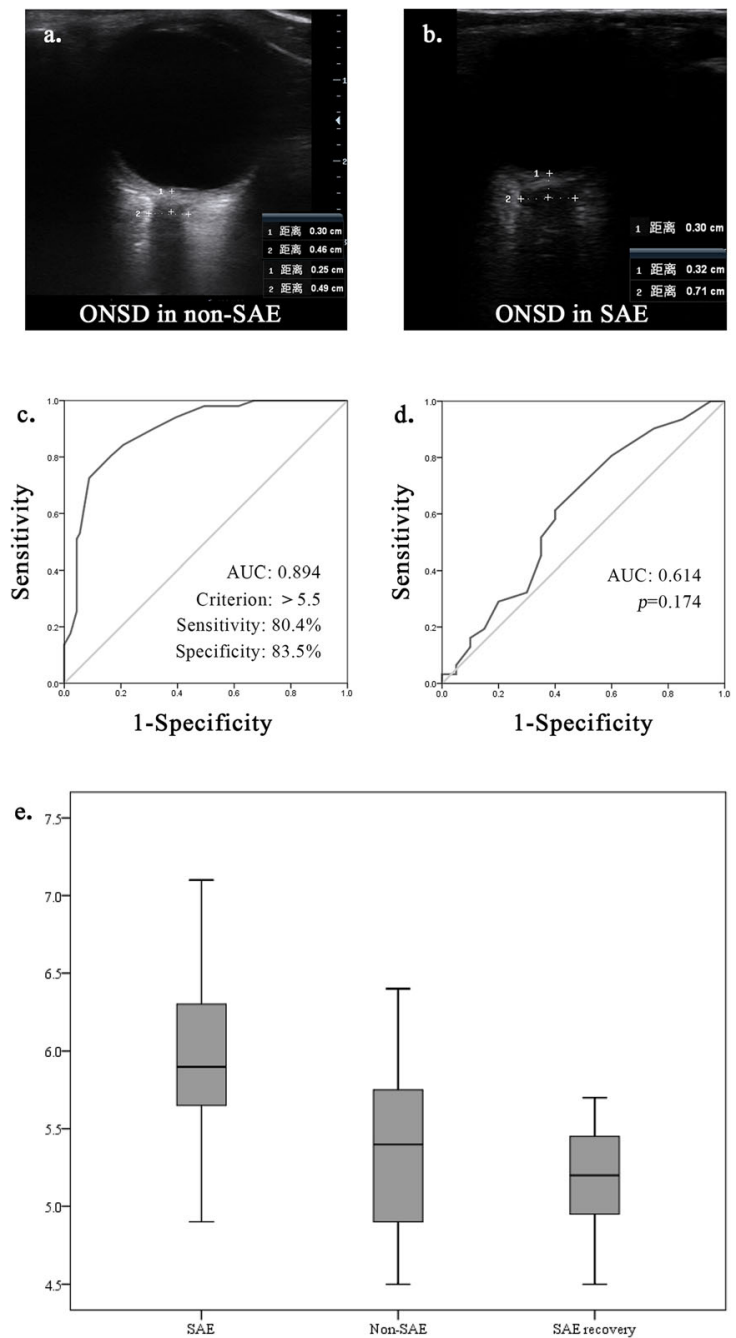

Fig. 1 a Ultrasonographic images of the optic nerve sheath in a patient with non-sepsis-associated encephalopathy. b Ultrasonographic images of the optic nerve sheath in a patient with sepsis-associated encephalopathy (SAE) (ONSD, optic nerve sheath diameter). c The receiver operating characteristic (ROC) curve for optic nerve sheath diameter (ONSD) to diagnose septic encephalopathy. $\mathbf{d}$ The ROC curve for ONSD to predict 28day survival in patients with sepsis-associated encephalopathy (AUC, area under the curve). e Comparison of the optic nerve sheath diameter among the three patient groups (SAE, sepsis-associated encephalopathy)

bedside angle $\left(0^{\circ} 5.5 \pm 0.5 \mathrm{~mm}\right.$ versus $\left.30^{\circ} 5.1 \pm 0.5 \mathrm{~mm}\right)$, even though only 18 patients were observed. In patients with SAE, the ONSD in deceased patients was slightly wider than that in surviving patients $(6[5.7,6.3] \mathrm{mm}$ versus $5.7[5.475,6.2] \mathrm{mm})$, but there was no statistical difference $(p=0.172$, Fig. $1 \mathrm{~d})$.

\section{Discussion}

We found that ONSD may be a new diagnostic tool for SAE. There is not enough evidence to show that patients with wider ONSD values have a higher risk of death, and ONSD cannot be used as an indicator for predicting the prognosis of patients with SAE. There are many influencing factors of ONSD; thus, it is necessary to measure it with a unified standard.

\section{Limitations}

ONSD may not be accurate enough, since it is the estimated value of ICP and not the definite value. Moreover, there may be many potential factors affecting the ONSD, which need to be further explored.

\section{Abbreviations}

ICP: Increased intracranial pressure; ONSD: Optic nerve sheath diameter; SAE: Sepsis-associated encephalopathy; CNS: Central nervous system; ICH: Intracranial hypertension; GCS: Glasgow Coma Scale; ROC: Receiver operating characteristic; AUC: Area under the curve

\section{Acknowledgements}

None.

Authors' contributions

All the authors contributed substantially to the work presented in this article. $\mathrm{ZYY}$ and $\mathrm{CHQ}$ designed the study, participated in the measurement of ONSD 
and the collection of case data, conducted the statistical analysis, and wrote the paper. SGZ, SHL, and TWS revised the article. All authors have read and approved the final manuscript.

\section{Funding}

This study was supported by the Scientific and Technological Innovation Leaders in Central Plains (Grant No. 194200510017), Provincial Ministry CoConstruction Project from the Medical Scientific and Technological Research Program of Henan Province (Grant No. SBGJ2018020), the "51282" Project Leaders of Scientific and Technological Innovative Talents from Health and Family Planning Commission in Henan Province (2016-32), and Science and Technology People-Benefit Project of Zheng Zhou (2019KJHM0001).

\section{Availability of data and materials}

All data generated or analyzed during this study are included in this published article and its supplementary information files.

\section{Ethics approval and consent to participate}

This study has been approved by the Scientific Research and Clinical Trial Ethics Committee of the First Affiliated Hospital of Zhengzhou University (Code 2020-KY-035).

\section{Consent for publication}

Not applicable.

\section{Competing interests}

The authors declare that they have no competing interests.

Received: 29 April 2020 Accepted: 8 May 2020

Published online: 18 May 2020

\section{References}

1. Chaudhry N, Duggal AK. Sepsis associated encephalopathy. Adv Med. 2014; 2014:762320.

2. Dubourg J, Javouhey E, Geeraerts T, Messerer M, Kassai B. Ultrasonography of optic nerve sheath diameter for detection of raised intracranial pressure: a systematic review and meta-analysis. Intensive Care Med. 2011;37(7):1059-68.

3. Robba C, Santori G, Czosnyka M, Corradi F, Bragazzi N, Padayachy L, Taccone FS, Citerio G. Optic nerve sheath diameter measured sonographically as noninvasive estimator of intracranial pressure: a systematic review and metaanalysis. Intensive Care Med. 2018;44(8):1284-94.

4. Singer M, Deutschman CS, Seymour CW, Shankar-Hari M, Annane D, Bauer M, Bellomo R, Bernard GR, Chiche JD, Coopersmith CM, et al. The Third International Consensus Definitions for Sepsis and Septic Shock (Sepsis-3). JAMA. 2016;315(8):801-10.

\section{Publisher's Note}

Springer Nature remains neutral with regard to jurisdictional claims in published maps and institutional affiliations. 